EPJ Web of Conferences 45, 01034 (2013)

DOI: $10.1051 /$ epjconf/20134501034

(C) Owned by the authors, published by EDP Sciences, 2013

\title{
Review of background oriented schlieren and development for ballistic range applications
}

\author{
Ardian B. Gojani ${ }^{1, a}$, Burim Kamishi ${ }^{2}$, and Shigeru Obayashi ${ }^{1}$ \\ 1 Institute of Fluid Science, Tohoku University, 2-1-1 Katahira, Aoba, 980-8577 Sendai, Japan \\ 2 Department of Physics, Faculty of Mathematical and Natural Sciences, University of Prishtina, Mother Theresa str., nn, \\ 10000 Prishtina, Kosovo
}

\begin{abstract}
Quantitative measurements of fluid flow can be achieved by flow visualization techniques, and this paper reviews and outlines background oriented schlieren, with emphasis on its performance: measurement sensitivity and uncertainty. Since the technique depends on cross-correlation, an assessment of image evaluation is also conducted. Background oriented schlieren is applied to two flows: shock reflection from a wedge in a shock tube, and natural cooling by convection. It is estimated that the technique can be applied to ballistic facilities.
\end{abstract}

\section{Introduction}

The idea of developing a quiet supersonic transport aircraft based on the Busemann biplane relies on the fact that wave drag which appears during supersonic fligh - and consequently, the intensity of the sonic boom, - can be reduced by a clever combination of aircraft elements. Details can be found in the article 4.19 of Liepmann and Roshko's book Elements of Gasdynamics, [1]. This concept is supported by theoretical considerations, as it historically originated and developed, and a wealth of numerical studies. A more recent parametric study is given in the work by Igra and Arad [2], and airfoil optimization in the work by $\mathrm{Hu}$ et al. [3]. A thorough numerical study with many references is presented in the Lecture Notes of Kusunose et al. [4].

The scarcity of experimental studies, on the other hand, is evidenced by the most recent review on the state of the study of supersonic biplane written by Kusunose et al. [5], where the only published experimental work on Busemann biplane prior to the ongoing work in Japan that started in mid 2000s, was conducted by A. Ferri, which was carried out in 1940s in a wind tunnel, and where aerodynamic forces were measured and flow visualization was done by using Toepler schlieren method. Applications of these two methods make the bulk of recent experiments, as well. Qualitative flow characteristics, mainly the effect of shock wave - expansion wave interference, are obtained indirectly by using schlieren visualization on wind tunnels, and the resulting images are later compared to CFD calculations, [69]. Smoke based visulaization in combination with force measurements has also been applied, with the aim to investigate the biplane behaviour at liftoff or nondesign conditions, $[9,10]$. The pressure distribution on the surface of the biplane has been experimentally measured by pressure and temperature sensitive paints, $[7,8,11]$, and in the work of Saito et al. some boundary layer effects were observed. Concluding remarks common to all these experiments are that, in general, flow field matches well with CFD predictions, but quantitative 3D (even 2D) measure-

\footnotetext{
a e-mail: gojani@edge.ifs.tohoku.ac.jp
}

ments are still required, and that biplane model supporting mechanisms in wind tunnels interefere with the overall flow. The second issue is addressed by experimenting with a free flight of a Busemann biplane model in a ballistic range, with schlieren visualization as the main diagnostic method, [12].

It is a normal development of this study to experimentally attempt quantitative flow field characterization in and around supersonic Busemann biplane. The closest to ideal measurements on a real model in free flight are measurements on a scaled model flying supersonically in a ballistic range. This experiment, then, requires the development of two independent tasks: (i) launching of the biplane model in a ballistic range facility, and (ii) measuring flow characteristics.

There is already a precedent with the first part, as reported by Toyoda et al. [12], while the ballistic range at IFS, Tohoku University is constantly used for launching projectiles up to hypersonic velocities. Thus, the operation of the ballistic range does not pose any challenges, if the model and flight conditions are provided.

For the measurement of flow characteristics, on the other hand, the best option lays with flow visualization. Direct temperature and pressure measurements by using gauges on a small model in a ballistic range are forbidingly difficult, while alternative options of temperature measurements by using imaging and spectroscopic methods (for example, thermal imaging, laser induced fluorescence, etc.) usually end up being expensive and complicated. Within two types of flow visualization, as categorized by Merzkirch in [13], marker methods (smoke visualization, particle image velocimetry, molecular tagging velocimetry, oil drops, etc.) are not applicable for the problem at hand, because of the complicated interaction of the model's flight and the seeded flow markers, or because they give only information about the flow properties on the surface of the wings. In optical flow visualization types of experiments (shadowgraph, schlieren, interferometry, speckle, etc.), light serves as the gauge of the refractive index or its variation in the flow field, and by virtue of direct relationship between re- 
fractive index and density, optical visualization yields density measurements. As already stated, schlieren methods have already been used even for Busemann biplane model in a ballistic range, but only qualitatively. Although schlieren method and its numerous modifications can give quantitative results, their implementation is very difficult - the difficulty being the rigorous demands on measurement precision and on high-quality optical components. With digital recording of light intensity, one can get some relative quantitative mearurements, but there still remains the problem of calibration for absolute measurements even for the amount of change of light intensity. The main obstacles to application of interferometry and its variations, e. g. MZI, holographic interferometry, shearing interferometry, etc. are the implementation difficulty and the requirements of specific coherent light sources, as well as high quality optical components.

The proposed alternatives for quantitative flow visualization based on pattern deflectometry are a combination of flow visualization methods and image analysis methods. Merzkirch in [14] defines moiré deflectometry for regular patterns. Instead, the proposed name pattern deflectometry would encapsulate all types of patterns (regular, random, colored, lined, dotted, naturally occurring, artificially produced, ...), and the -metry part would ensure of the quantitative nature of the method. In applications to gas dynamics problems, this method is refered as background oriented schlieren (BOS), while other names, such as synthetic schlieren, grid schlieren, etc., are also used for techniques with minor variations. The quantiative results extracted depend heavily on image analysis methods developed for computer vision $[15,16]$.

\section{Principles of BOS}

The fundamentals of BOS technique have been outlined in references [17] and [18], and a broad assessment is given in [19]. BOS falls in the category of pattern deflection techniques, similar to grid schlieren and moiré deflectometry, as opposed to standard schlieren, which records the variations of light intensity on the recording plane. Thus, the principle of BOS measurement lays in the difference in a pattern imaged through a test fluid at two different states: the reference image is taken without, and the BOS measurement image is taken with the disturbance that is to be evaluated present in the fluid. The disturbance, e. g. a vortex, a heat wave or a shock wave, causes local changes of fluid's density, resulting in transient changes of the refractive index of that fluid at the points through which the disturbance is propagating. Hence, the imaging light beams passing through the disturbance will deflect, resulting in the shift of some features that are being imaged. The angle of deviation, and consequently the value of the refractive index, is encoded in the difference between the reference and measurement images, and can be extracted, for example, by analyzing images with cross-correlation algorithms, [20], [21]. Thus, in its simplest form, BOS is a lineof-sight integrating technique that gives the $2 \mathrm{D}$ projection of the refractive index of the fluid.

BOS has been applied to numerous types of flows in different conditions. Diagnostics of flows in supersonic and hypersonic facilities by BOS has been carried out in references [22-26], while in references [27-29] BOS has been used in the outdoors, with a large field of view. Several improvements of the technique are proposed, mainly in obtaining 3D measurements by tomographic imaging, [30$32]$, or by increasing the accuracy through colored backgrounds, [33].

A BOS measurement procedure consists of two parts: (i) image recording, and (ii) image evaluation.

\subsection{Image recording}

In the image recording part, reference and measurement images are taken at two instances, as illustrated in figure 1. This figure shows a typical BOS setup consisting of a structured background $B$, the test section under investigation $T$, also referred to as the phase object, transfer channel, density field, etc., the objective lens $L$ with focal length $f$, and the image recording sensor $I$. When the reference image is being recorded, a feature from the background located at point $\mathbf{B}$ is imaged in the point $\mathbf{I}$. Introduction of the fluid flow with variable refractive index $n(\mathbf{r})$ will deflect the beam for the angle $\varepsilon$, thus the image of point $\mathbf{B}$ now will be shifted for $\Delta_{i j}$ to point $\mathbf{J}$. This effect will be recorded only if the shift is larger than the linear dimensions of the pixel $\ell_{p x}$, that is, if $\Delta_{i j} \geq \ell_{p x}$. This condition represents the detection limit of the system.

A light ray propagating through the test section in the direction of unit vector $\mathbf{s}$, is bent due to the variation of the refractive index. The reciprocal of the local radius of curvature $R$ is given by [34]

$$
\frac{1}{R}=\frac{\mathrm{d} \mathbf{s}}{\mathrm{d} s} \cdot \nabla \ln n
$$

On the other hand, from geometric considerations,

$$
\mathrm{d} \varepsilon=\frac{\mathrm{d} s}{R} .
$$

The total angle of deflection is found by integrating along the entire path through which the refractive index varies, thus

$$
\varepsilon=\int_{w} \nabla \ln n \mathrm{~d} s \approx \frac{w}{n_{0}} \nabla n,
$$

where $n_{0}$ is the refractive index of the air and $w$ is the width of the test section, or the length of the inhomogeneity in $n(\mathbf{r})$. The refractive index is related to density $\rho$ through the Gladston-Dale equation, $n-1=K \rho$, where $K=2.23 \times$ $10^{-4} \mathrm{~m}^{3} \mathrm{~kg}^{-1}$, therefore it is straightforward to express the density change in the fluid as a function of the angle of deflection.

Angle of deflection $\varepsilon$ and feature shift in the image plane $\Delta_{i j}$ are related by equation

$$
\Delta_{i j}=M s_{t} \varepsilon,
$$

where $M$ is the transverse magnification of the system. Sensitivity, which for the case at hand is defined as $S=$ $\Delta_{i j} / \varepsilon$, then is

$$
S=M s_{t}
$$

Since the lens is focused on the background, the measurement point $\mathbf{T}$ will be imaged into a disk, due to blur. Furthermore, regardless of the diffraction limit, but considering that the image sensor is a rectangular grid of pixels each integrating the features acquired from the incident 


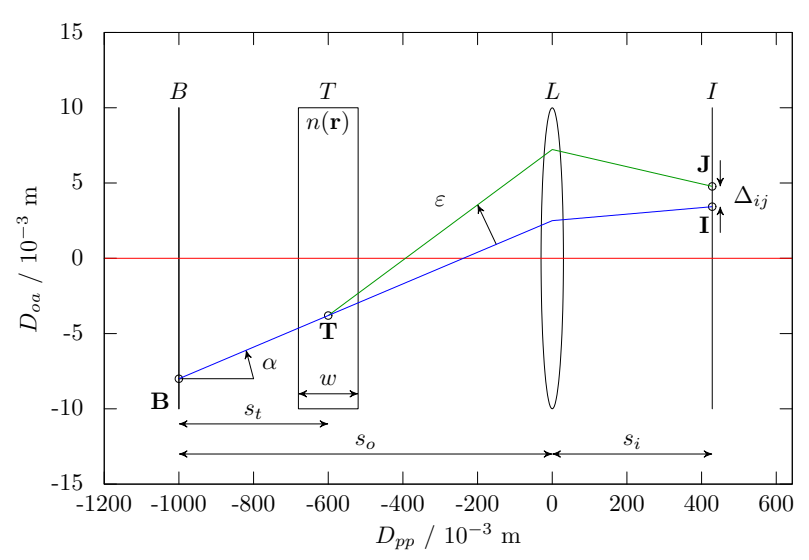

Fig. 1. A meridional plane of a BOS setup: $B$ - the background, $T$ - the test section with the variable refractive index $n(\mathbf{r})$ and width $w, L$ - the imaging lens focused on the background, $I-$ the image plane, coplanar with the recording sensor. Distances between these planes and the angles of the light rays are also given, while in axes $D_{p p}$ is the distance from the principal plane (the lens) and $D_{o a}$ is the distance from the optical axis. The blue line indicates the light ray during the imaging of the reference image, while the green line illustrates the deflection of the ray in the test section and shows the ray position during the measurement image.

cone of light on it, even a point in the object plane is not imaged into a point, but into a square the size of a pixel, resulting in a discretized image of the object plane, as illustrated in figure 2. For a given circle of the least confusion $\Delta_{t}$, the size of the recorded feature on the background will be $\delta_{b}=\Delta_{t} / M$, while the diameter of the physical spot at the test section is given by

$$
\delta_{t}=\frac{M}{M+1} \frac{s_{t}}{f_{\#}}+\frac{\Delta_{t}}{M},
$$

where $f_{\#}$ is the f-number of the lens. Equation 6 presents the spatial resolution of the BOS system obtained during the image recording stage. It also justifies neglection of the shift of the light ray inside the test section, thus allowing for the approximation that the light rays enter and exit the test section at the same distance from the optical axis.

Equations 5 and 6 present the sensitivity and uncertainty of a BOS optical setup, respectively. Their plot as a function of the optical magnification $M$ and the distance $s_{t}$ between the imaged background and the flow field is given in figure 3. This plot presents the experiment design space for unit values of $f_{\#}$ and $\Delta_{t}$. In practical implementations, $f_{\#}=5.6-40$, and $\Delta_{t}=3-4 \times \ell_{p x} \approx 10-20 \mu \mathrm{m}$.

\subsection{Image evaluation}

The result of image recording are two images, $I_{r}$ and $I_{m}$, i. e. reference and measurement images as vectors of grayscale values. In the image evaluation step it is required to extract $\Delta_{i j}$, which is achieved by cross-correlation. In crosscorrelation, a subset of one of the images (representing the interrogation window $W$ ) is defined, and then its grayscale values are compared for similarity to grayscale values of all equal subsets of the other image. In this case, the vectors are multiplied and the most similar subsets yield the highest product (maximal value being 1). The product, known

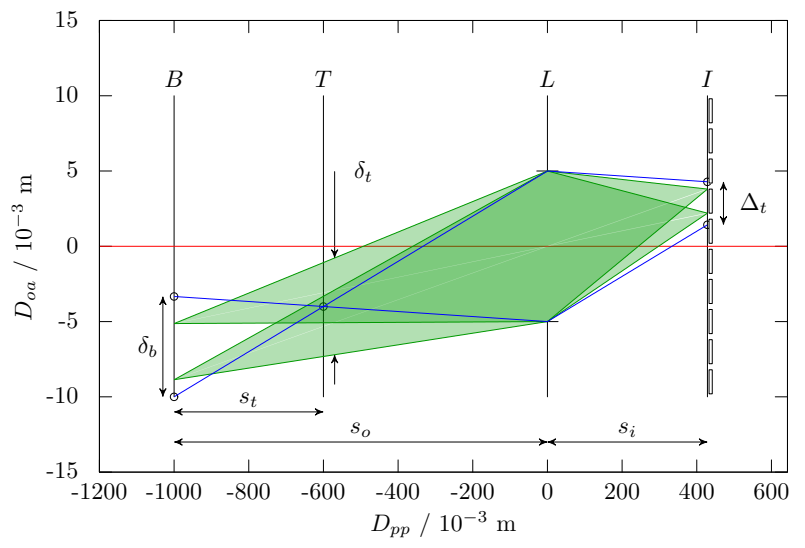

Fig. 2. Circle of least confusion $\Delta_{t}$ and spatial resolution $\delta_{t}$ per pixel. $\delta_{b}$ is the smallest feature on the background that can be resolved in imaging, effectively determining the diameter of a dot. In axes, $D_{p p}$ is the distance from the principal plane (the lens) and $D_{o a}$ is the distance from the optical axis.

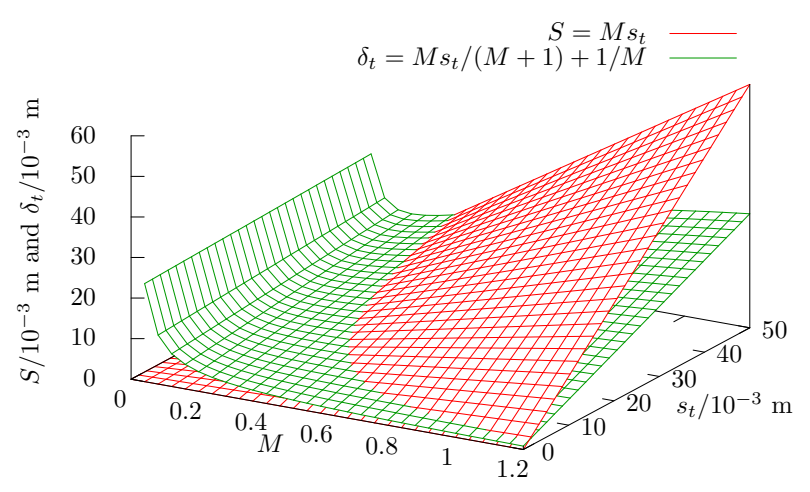

Fig. 3. Surface plot of the sensitivity $S$ (red surface, equation 5) and uncertainty $\delta_{t}$ (green surface, equation 6) of a BOS system for unit values of $f_{\#}$ (f-number of the lens) and $\Delta_{p x}$ (linear dimension of the pixel size). For small magnifications, uncertainty plays the determining role in adjusting the system, while for large magnifications, the system can be made quite sensitive.

as the cross-correlation coefficient, is

$$
\begin{aligned}
c(p, q)= & \sum_{h} \sum_{v} W(h, v)\left[I_{r}(h, v)-\left\langle I_{r}\right\rangle\right] \times \\
& \times W(h+p, v+q)\left[I_{m}(h+p, v+q)-\left\langle I_{m}\right\rangle\right],
\end{aligned}
$$

where $\left\langle I_{r}\right\rangle$ and $\left\langle I_{m}\right\rangle$ are the average values of reference and measurement images over window $W$, and $h$ and $v$ are the horizontal and vertical pixel locations. The summation is done over all pixel count values, with the largest number equal to the pixel count of the camera $N_{p x}=H \times V$. $p$ and $q$ are trial displacement components. Thus, the result of the cross-correlation is a spatial displacement vector $c(p, q)$, which relates the correlation peaks. Applying a three point Gaussian peak detection scheme, this vector can be determined with an accuracy better than 0.1 pixel (in some implementations, down to 0.01 pixel). The magnitude of $c(p, q)$ is the amount of pixel shift due to light ray deflection of a background pattern projected (imaged) in the image plane, $\Delta_{i j}$.

Displacement vector obtained by image analysis is influenced by IW, pattern size, spatial frequency of pattern texture, and gradients within IW, which are responsible for error sources such as peak locking effect, pattern smoothen- 
ing from non uniform image blur, etc. Some of these effects are studied by numerical experiments in synthetic images, in particular the effect of the IW, the background dot size, dot density, and the introduction of blur. Half of a $128 \times 128$ pixels synthetically generated reference image of randomly distributed dots is shifted for a number of pixels, giving the simulated measurement image. This corresponds to the treatment of the image with the step function $H[N]$, where

$$
H[N]=\left\{\begin{array}{l}
0 \text { for } N<0, \\
1 \text { for } N \geq 0,
\end{array}\right.
$$

with $N=H / 2$. In other words, the image is sliced in two equal parts, with the right side (pixels in horizontal locations 64 and higher) slided for one or more pixels to the left. Then, the simulated reference and measurement images are cross-correlated. The effects are observed by the changes on the step function response width (SFWR), as defined in [35].

Image evaluation with different IW shows that the smaller the IW the closer the evaluated jump is to the real step function, as shown in figure 4 . In this investigation, the simulated images had a dot the size of a pixel, and the image coverage by dots was $50 \%$. The spread and gradual increase of the discontinuity means that there can not be an independent shift vector within the length it takes the evaluated pixel shift value to jump. Therefore, the evaluated spatial resolution for IW $=32$ pixel is about 38 pixel, and for IW $=8$ it is about 16 pixels. Multipass evaluation with successively smaller IW did not show any improvement in the evaluation of the jump, while it gave erroneous (fluctuating) values for the amount of the pixel shift.

The preparation of the background involves determination of the dot size, frequency (in terms of the number of dots in the field of view), and coverage. While in experiments with natural backgrounds the experimenter has no control over the background features, in experiments under laboratory conditions (e. g. shock tube or wind tunnel experiments), the experimenter can prepare a background that optimizes the measurement based on the camera specifics and the field of view. Figure 5 shows the effect of the dot size in the resolution, where dot sizes $\delta_{b}$ equal to one and four pixels are compared after images are treated by a one pixel step function. The evaluation does not show any large effect on the resolution, but it does give different values for the pixel shift. For the case with $\delta_{b}=4$ pixels, the effective shift of the image corresponds to a quarter of a dot, hence some pixels are not recorded as shifted. This situation appears in synthetic image evaluations, because these images have a well defined binary structure. In experiments, an image of a binary background results in a grayscale image with spread histogram peaks around the binary values. This leads to more accurate results during evaluation. Figure 6 shows the effect of dot density, which is defined as the number of dots per unit area of the field of view projected in the total image area. Maximal dot density, 50\%, means that half of the image is covered by dots, and the minimal density simulated ( $5 \%$ coverage with dots) is mainly a white featureless background. As previously, the image is treated to a one pixel shift and evaluated with IW $=8$ pixel. Virtually, there is no difference in evaluating images with dot density of higher than $20-25 \%$, but pixel shift evaluation artifacts start showing for images with lower number

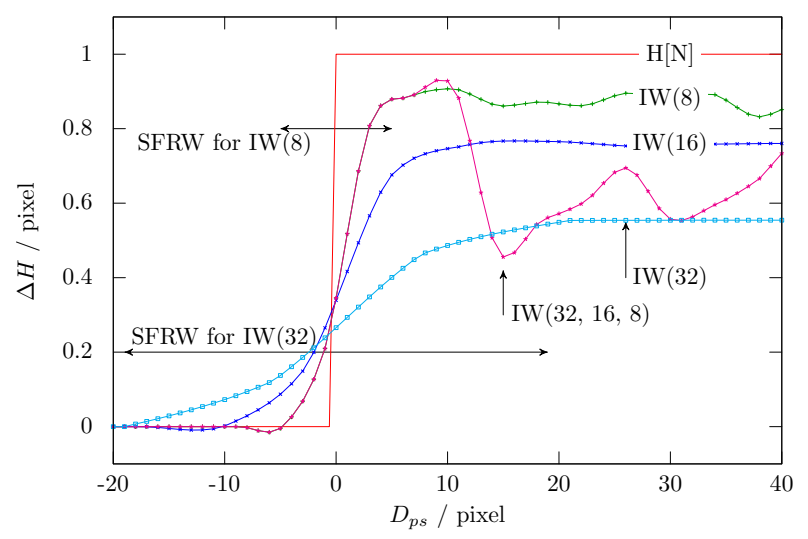

Fig. 4. The effect of the size of the interrogation window on the cross-correlation evaluation. $D_{p s}$ is the distance from the location of pixel shift, $H[N]$ is the step function, SFRW is the step function response width, and $\operatorname{IW}(a)$ is the interrogation window, with $a$ being its value in pixels. For IW(32, 16, 8), multipass evaluation was carried out.

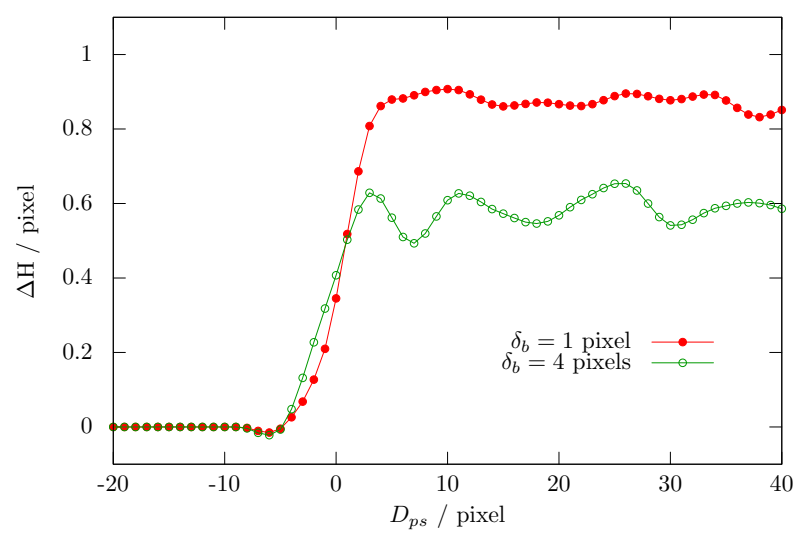

Fig. 5. The effect of the background dot size on evaluating pixel shift.

of dots. This result is in agreement with the previously published requirement that an IW should have at least four to five dots, each covering 2 pixels.

The effect of the blur is investigated by treating the synthetic measurement image with a Gaussian blur of radius of 2 pixels, and the result is shown in figure 7. In this case, the image was shifted for five pixels and the interrogation window was 16 pixels. The effect of the blurring in SFRW is negligible, but this is not so for the determination of the amount of pixel shift. The blurring of the image has the effect of reducing this value for about $10 \%$ and introducing fluctuations in its behaviour, thus yielding a lower signalto-noise $(\mathrm{S} / \mathrm{N})$ ratio. This effect comes about because, as compared to the binary image, the blurred image is populated by all possible grayscale values and pattern's spatial frequency is not conserved.

\section{Experiments}

Two examples of application of BOS to experiments will be given: one for the reflection of a shock wave from an inclined plane (wedge) in a shock tube, and one for the determination of the temperature field during the natural convective cooling. 


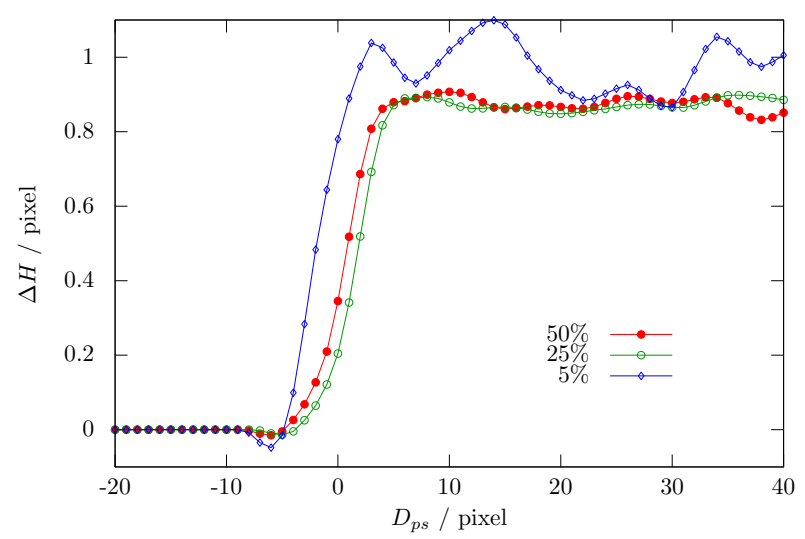

Fig. 6. The effect of the dot distribution density on evaluating pixel shift.

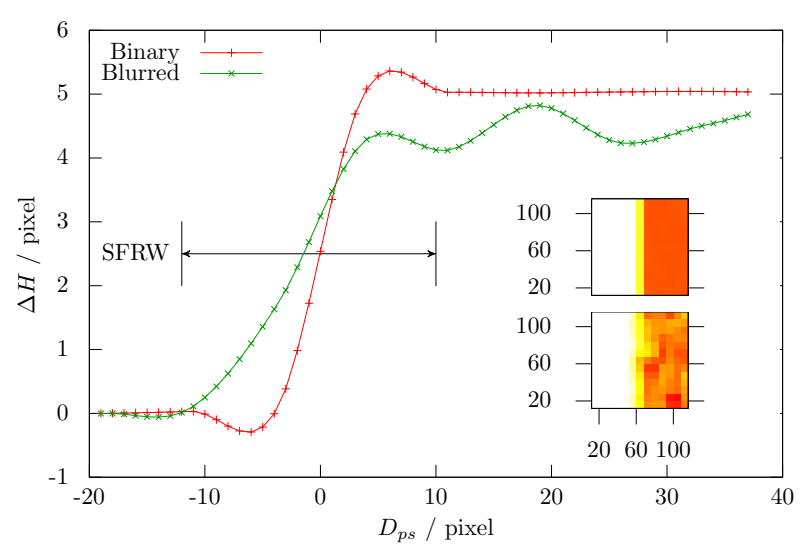

Fig. 7. Pixel shift evaluation by cross-correlation ( $\mathrm{IW}=16$ pixels) of binary (inset, up) and Gaussian blurred (inset, down) synthetic images with dots of one pixel, shifted for 5 pixels. Tics in insets are image pixels. Again, SFRW is virtually the same for both cases, but the $\mathrm{S} / \mathrm{N}$ changes for the worst in the case of blurred images.

\subsection{Shock reflection}

A BOS experiment for the investigation of shock diffraction and reflection from a wedge (inclined plane) in a shock tube was conduced, with the aim of testing BOS capabilities, because the passage of a planar shock wave is a well studied and documented phenomenon, thus it can serve as a benchmark test. The shock tube was run with air at $P_{1}=0.10 \mathrm{MPa}$ as the driven gas and nitrogen $\mathrm{N}_{2}$ at $P_{4}=$ $0.35 \mathrm{MPa}$ as the driver gas. The model was a stainless steel wedge with the base attached to the top of the shock tube, effectively creating an inclined plane with inclination of $49^{\circ}$. Shock propagation was monitored by three Kistler 603B pressure transducers, which also sent the triggering signal for the image recording system. Timing of the experiment was controlled by a combination of an oscilloscope and a pulsed delay generator. Shock Mach number for all experiments was 1.3 , and since the effective field of view was $220 \mathrm{~mm} \times 150 \mathrm{~mm}$, a high speed camera was needed for freezing shock's motion. For this reason, two types of cameras were used:

- Shimadzu HPV-1 camera with $312 \times 260$ pixel count and time resolution of $0.5 \mu \mathrm{s}$, capable of taking $100 \mathrm{im}-$ ages with a maximal frame rate of $1 \mathrm{Mfps}$. The camera sensor is of the IS-CCD type, with a linear dimension of pixel's light collecting area of about $50 \mu \mathrm{m}$;

- Imacon DRS 200 camera with $1200 \times 980$ pixel count and linear dimension of pixel size approximately 6.5 $\mu \mathrm{m}$. This camera has 7 channels, each being capable of taking two images with the fastest interframe of 1 $\mu \mathrm{s}$, thus resulting in a total of 14 images. Since all 7 channels are independent, their respective interframes can be adjusted freely. The minimal exposure time of the camera is $5 \mathrm{~ns}$, which is faster than the required and used $1 \mu$ s time resolution.

The main differences between these two cameras come from their pixel count, with Imacon having a $10 \times$ better characteristic, and the pixel area, with the Shimadzu one having a $10 \times$ larger area. The background used in the shock tube experiments was a white sheet with randomly distributed square dots of $1 \mathrm{~mm}$ side length. This background was illuminated by a xenon flashlamp that has pulse duration longer than $1 \mathrm{~ms}$. Although in both experiments the background was a binary image, that is with only white and black areas, the recorded image was an 8 bit grayscale digital file.

\subsection{Natural convection}

The steady temperature field was achieved by a cylindrical radiative heater with diameter of $10 \mathrm{~mm}$, length of 150 $\mathrm{mm}$ (corresponding to $w$ in figure 1), and power of 100 $\mathrm{W}$, placed perpendicularly to the background. Since the temperature field changed slowly with time, the requirements on the temporal resolution were minimal, so a standard DSLR Pentax K-5 (digital single-lens reflex) camera was used. This camera has a sensor of $23.6 \mathrm{~mm} \times 15.8$ $\mathrm{mm}$ and pixel count of $4928 \times 3264$ pixels, hence a linear pixel size of about $5 \mu \mathrm{m}$. The objective lens had a focal length of $300 \mathrm{~mm}$ and experiments were done with aperture of $f / 32$. Small apertures were used in order to obtain longer depths of field. Illumination was achieved by backlighting the printed background with a xenon flashlamp, and the camera exposure times were 1/180 s. Several types of backgrounds were generated by printing a random dot pattern in an A4 paper. Setup distances were adjusted to achieve an optical magnification of the system $M=0.1$, with 30 pixels imaging $1 \mathrm{~mm}$ of the field of view. The temperature was simultaneously monitored by 2 thermocouples with temperature resolution of $0.1{ }^{\circ} \mathrm{C}$.

\section{Results}

\subsection{Instrumentation - image quality}

The speed with which a fluid flows determines the shutter speed (exposure time) that the camera needs to operate with, and this proves to be one of the most demanding factors in choosing an image sensor. Shock waves in a shock tube, for example, can be imaged only with scientific grade high-speed cameras, such as Imacon DSR200 or Shimadzu HPV-1, which are capable of imaging at times shorter than $1 \mu \mathrm{s}$. Slow flows that can reach a pseudosteady state, such as slow cooling by natural convection, can be imaged with standard DSLR cameras. In these later cases, 
exposure time is not a limiting factor, because it can be large enough to reach an average value of temperature reading smaller than the measurement uncertainty, but still be orders of magnitude smaller than the temperature measurement steps. Thus, one would be free to choose a camera with a high pixel count or a large sensor size, so that the image detail is satisfactory for precise measurements.

Images shown in figure 8 are from both types of high speed cameras and show a detail of the same background imaged through the test section of a shock tube. If one considers the sensitivity of a BOS setup based on the geometry of the layout and the size of the image sensor, Imacon camera would be preferable to Shimadzu, for two reasons: larger pixel count and smaller pixel size. But, despite the facts that the shown field of view is the same and that the images were taken with the same lens as well as under the same illumination, obtained images are quite different, which fundamentally comes about due to the different quantum efficiencies of the respective image sensors. A dramatic outcome of this difference is that the direct (without any processing) evaluation with cross-correlation of images captured by Imacon camera could not give any meaningful results, while images captured by Shimadzu did, as illustrated later. The difference can be explained through different response to luminance of the image sensors and the contrast values of the output file. Both cameras have a sensor with 10 bit dynamic range and give comparable dark images, but the histogram of the measurement images, shows that the Shimadzu camera produces a better contrast.

Since the images in BOS are of randomly scattered dots, that is structureless distribution of black and white dots, it is of prime importance that cameras yield high image quality. One way of determining the quality of images is by measuring the universal image quality index, as proposed in [36], which in a modified form is

$$
Q_{\text {mod }}=Q_{L} Q_{C}=\frac{2\langle x\rangle\langle y\rangle}{\langle x\rangle^{2}\langle y\rangle^{2}} \frac{2 \sigma_{x} \sigma_{y}}{\sigma_{x}^{2}+\sigma_{y}^{2}},
$$

where $x$ is the captured image, $y$ is an ideal to which $x$ is being measured, $\langle x\rangle$ and $\langle y\rangle$ are their respective average values, and $\sigma_{x}$ and $\sigma_{y}$ their standard deviations. The first term in equation $9, Q_{L}$, corresponds to the comparison of the luminance between images, the second term, $Q_{C}$, describes the comparison of the contrast between the images. The closer the image $x$ is to an ideal $y$, the closer these values will be to 1 . Overall, $Q_{\text {mod }}$ quantifies the response of the camera sensor and its quantum efficiency in a simple manner, giving a value that allows for a judgement whether a camera can give a file that can be evaluated by cross-correlation. Values of $Q_{\text {mod }}$ for the cameras used in these experiments are given in table 1 .

\subsection{Shock reflection}

Results of cross-correlation for the shock reflection experiment are shown in figure 9, where the magnitude map of dot displacement vector shift is plotted. The interrogation window for this evaluation was 8 pixels, thus giving a resolution of 10 pixels. Since the optical magnification was 0.1 , the measurement uncertainty then is $5 \mathrm{~mm}$. As already stated when the role of the interrogation window was discussed, its large value results in the spill of the pixel shifts
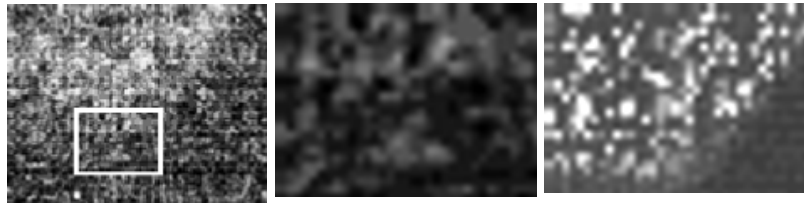

Fig. 8. Images on the left and on the center - the latter being the framed part of the former, - are taken with Imacon DSR200 camera, and that on the right with Shimadzu HPV-1. The left and right images show the same field of view, while the one on the center and that on the right have the same pixel count.

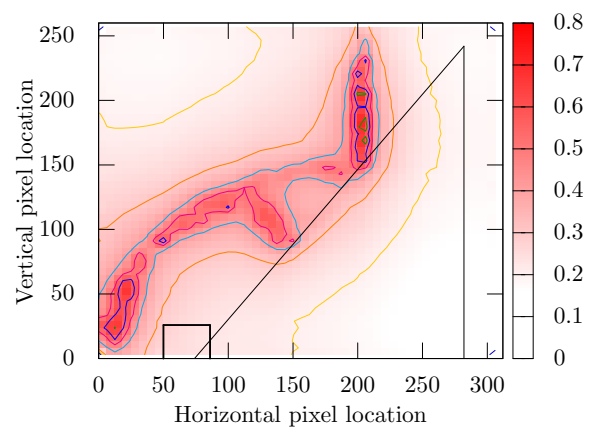

1

Fig. 9. Background oriented schlieren result for the shck reflection from a wedge (black full lines), shown as a magnitude map of vector shift, with pixel locations in coordinates and pixel shift amount in the magnitude bar. The small rectangle starting at pixel $(50,0)$ is the part of the image given in figure 8 .

inside the wedge. Nevertheless, some clear features of the phenomena are observed, such as the shock wave, its reflection, and the sonic domain.

\subsection{Natural convection}

While these results for density gradient measurement behind a shock wave in a shock tube are very coarse, mainly due to the low pixel count of the high speed camera, the results of BOS applied to natural convection show fine detail, as given in figure 10. In the figure, contour lines border the zones with 0.5 pixel shift, which corresponds to temperature difference of 30 degrees. Comparison of temperature evolution by BOS, thermocouple readings, and according to Newton's law of cooling shows a satisfactory agreement. BOS imaging was done with an extra large pixel count camera (16 megapixels), which had several benefits: a dot covered $8 \times 8$ pixels, the dots had five distinct grayscale values, IW was $16 \times 16$ pixels. For the sake of computational speed, the image was reduced in size 4 times per direction, giving two pixels per dot. The dot density was $50 \%$ and the magnification of the setup was 0.15 , with 30 pixels covering $1 \mathrm{~mm}$ of the field of view. Since cross-correlation in BOS can determine pixel shifts down to $0.01 \mathrm{px}$, one can conclude that the measurement sensitivity of BOS can be considered to be of the same order as that of the thermocouples, namely $0.6^{\circ}$ for BOS with $0.1^{\circ}$ for thermocouples. 


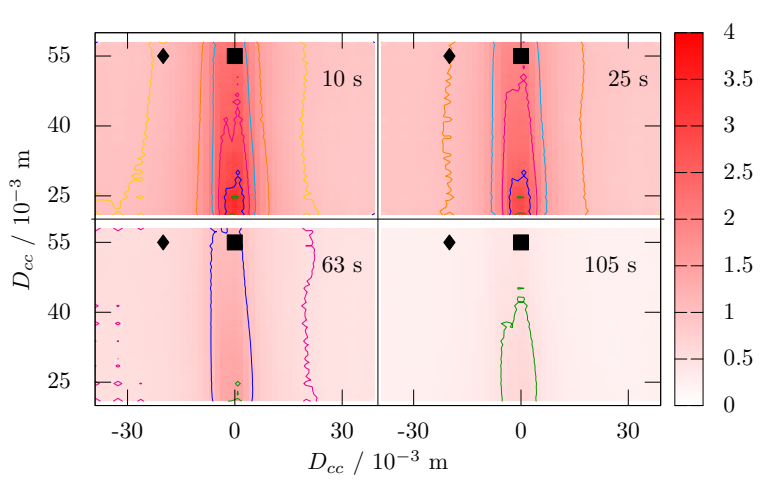

1

Fig. 10. BOS results for cooling by natural convection, taken at four different times: $10 \mathrm{~s}, 25 \mathrm{~s}, 63 \mathrm{~s}$, and $105 \mathrm{~s}$ after the heater was turned off (times noted in the upper right courner of each map). $D_{c c}$ is the distance from the center of the heating cylinder. Thermocouple positions are noted by the symbols $\mathbf{\square}$ and $\downarrow$. Coordinates give the distance from the center of the heat source, in $\mathrm{mm}$, and the magnitude bar gives the pixel shift. Contour lines correspond to borders between zones with pixel shift diference of 0.5 pixel.

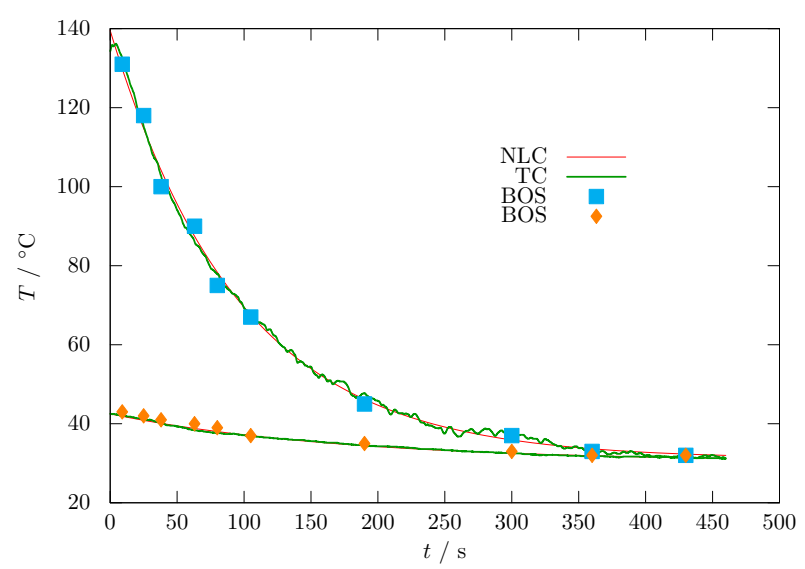

Fig. 11. Quantitative BOS result for cooling by natural convection, and the comparison of BOS data to thermocouple (TC) readings and Newton's law of cooling (NLC). Here, temperature $T$ is given as a function of time $t$.

Table 1. Luminance and contrast image quality indices for high speed cameras (Imacon, Shimadzu) and standard DSLR cameras (Pentax K-5).

\begin{tabular}{rcc}
\hline & QL & QC \\
\hline Imacon DSR200 & 0.18 & 0.07 \\
Shimadzu HPV-1 & 0.62 & 0.54 \\
Pentax K-5 & 0.99 & 0.71 \\
\hline
\end{tabular}

\section{Prospects for ballistic range experiments}

In applying the BOS technique for ballistic range measurements, one should take into consideration the small size of the model and its fast velocity. Current research predicts that the optimal cruise flight will be with Mach number about 1.7. The small size of the model would allow for a relatively large magnification: for a $50 \mathrm{~mm}$ model, and considering double the size of the field of view, $M \approx 0.3$.
But, this would require a very fast shutter speed. During the exposure time of $1 \mu \mathrm{s}$, a model flying at speed $\approx 600$ $\mathrm{m} \cdot \mathrm{s}^{-1}$ will traverse more that $0.5 \mathrm{~mm}$, limiting the field of view imaged by a pixel in at least $1 \mathrm{~mm}$ (or, alternatively, limiting the minimal size of the background dot at least at $1 \mathrm{~mm}$, for a dot per pixel). As a consequence, the field of view would appear quite coarse.

An alternative is the use of short light pulse for imaging (e.g. nanosecond white light flash system, or a pulsed laser with destroyed coherence) in combination with a high resolution camera. Increased magnification would allow for sensitive measurements, as well as for the decrease of the distance between the background and the test section, $s_{t}$, which would make possible having the entire test section in focus by using large depths of field.

Another improvement in spatial resolution of the measurement would be using single-pixel correlation, although this would require a large number of experimental images under the same conditions.

\section{Conclusions}

The development of background oriented schlieren (BOS) technique is advanced by assessing several factors that influence the extraction of quantitative data, be it in the experimental or image evaluation stage. The sensitivity and uncertainty of a BOS setup was obtained, as well as the image quality index was introduced to assess the instrumentation. This assessment would not only assist with the judgment of the image quality and whether the image would yield meaningful results after cross-correlation, but also would be beneficial for diagnostics of the faulty arrangements (illumination or contrast).

Investigation of the geometrical arrangement of the instruments used for BOS visualization reveal that arbitrary sensitivity and resolution can be achieved, but, these specifications, though, are later deteriorated by image evaluation. Uncertainties related to the point of measurement are influenced by the interrogation window, defining the spatial resolution of the measurement, while image blurring influences the determination of the pixel shift.

In accordance to previous studies, investigation of background featurres showed that a good cross-correlation evaluation requires at least four to five dots for an interrogation window, with a dot covering two pixels.

BOS technique was applied to two types of flows, with variable success. Shock reflection from a wedge was visualized, albeit only qualitatively. Several features were observed, such as the reflected shock, and probably the acoustic region behind the refelection. In the case of natural convection, the results had a quantitative nature, comparable to the results obtained with thermocouples.

Present state of BOS allows for its application to ballistic range experiments. Although quantitative results are anticipated, the current high speed cameras have a rather low pixel count, thus two alternatives are proposed: using short pulse powerfull light source, and carrying image evaluation by single-pixel correlation. 


\section{Acknowledgment}

This work was supported by Global Center of Excellence Program "World Center of Education and Research for Transdisciplinary Flow Dynamics," Tohoku University, Japan.

\section{References}

1. H. W. Liepmann and A. Roshko, Elements of Gasdynamics (John Wiley and Sons, New York 1957)

2. D. Igra and E. Arad, Shock Waves 16, 269-273 (2007)

3. R. Hu, A. Jameson and Q. Wang, AIAA paper 20111248, (2011)

4. K. Kusunose, K. Matsushima, S. Obayashi, T. Furukawa, N. Kuratani,Y. Goto, D. Maruyama, H. Yamashita and M. Yonezawa, Aerodynamic Design of Supersonic Biplane: Cutting Edge and Related Topics The 21st Century COE Program, International COE of Flow Dynamics Lecture Series, volume 5 (Tohoku University Press, Sendai 2007)

5. K. Kusunose, K. Matsushima and D. Maruyama, Progress in Aerospace Sciences 47, 53-87 (2011)

6. N. Kuratani, T. Ogawa, H. Yamashita, M Yonezawa and S. Obayashi, AIAA paper 2007-3674, (2007)

7. K. Saito, H. Nagai, T. Ogawa and K. Asai, Proc. of 22nd ICIASF, 1-8 (2007)

8. N. Kuratani, M. Yonezawa, H. Yamashita, S. Ozaki, T. Ogawa and S. Obayashi, Proc. of 26th ICAS, 20082.4ST (2008)

9. H. Kawazoe, S. Abe, T. Matsuno, G. Yamada and S. Obayashi, Proc. of 27th ICAS, 2010-2.10ST2 (2010)

10. M. Kashitani, Y. Yamaguchi, Y. Kai, K. Hirata and K. Kusunose, AIAA paper 2008-349, (2008)

11. H. Nagai, S. Oyama, T. Ogawa, N. Kuratani and K. Asai, Proc. 26th ICAS, 2008-3.7.5 (2008)

12. A. Toyoda, M. Okubo, S. Obayashi, K. Shimizu, A. Matsuda and A. Sasoh, AIAA paper 2010-873, (2010)

13. W. Merzkirch, Flow Visualization (Academic Press, New York, 1987)

14. W. Merzkirch, in Springer Handbook of Experimental Fluid Mechanics, editors C. Tropea, A. L. Yarin and J. F. Foss, (Springer, Berlin 2007)

15. D. A. Forsyth and J. Ponce, Computer Vision: a Modern Apporach, (Prentice Hall, New York 2002)

16. B. Jähne, Digital Image Processing, (Springer, Berlin 2002)

17. H. Richard and M. Raffel, Meas. Sci. Technol. 12, 1576-1585 (2001)

18. G. E. A. Meier, Exp. Fluids 33, 181-187 (2002)

19. E. Goldhahn and J. Seume, Exp. Fluids 43, 241-249 (2007)

20. M. Sjödahl, Appl. Opt. 36, 2875-2885 (1997)

21. M. Raffel, C. E. Willert, S. T. Wereley and J. Kompenhans, Particle Image Velocimetry, (Springer, Berlin 2007)

22. F. Klinge, T. Kirmse and J. Kompenhans, Proc. of PSFVIP-4, F4097 (2003)

23. L. Venkatakrishnan and G. E. A. Meier, Exp. Fluids 37, 237-247 (2004)

24. D. Ramanah, S. Raghunath, D. J. Mee, T. Rösgen and

P. A. Jacobs, Shock Waves 17, 65-70 (2007)

25. T. Mizukaki, Shock Waves 20, 531-537 (2010)
26. M. Ota, K. Hamada, H. Kato and K. Maeno, Meas. Sci Technol. 22, 104011 (2011)

27. M. Raffel, H. Richard and G. E. A. Meier, Exp. Fluids 28, 477-481 (2000)

28. O. K. Sommersel, D. Bjerketvedt, S. O. Christensen, O. Krest and K. Vaagsaether, Shock Waves 18, 291-297 (2008)

29. M. J. Hargather and G. S. Settles, Exp. Fluids 48, 5968 (2010)

30. K. Kindler, E. Goldhahn, F. Leopold and M. Raffel, Exp. Fluids 43, 233-240 (2007)

31. B. Atcheson, W. Heidrich and I. Ihrke, Exp. Fluids 46, 467-476 (2009)

32. L. Venkatakrishnan and P. Suriyanarayanan, Exp. Fluids 47, 463-473 (2009)

33. F. Sourgen, F. Leopold and D. Klatt, Opt. Laser Eng. 50, 29-38 (2012)

34. M. Born and E. Wolf, Principles of Optics (Cambridge University Press, Cambridge 1999)

35. C. J. Kähler, S. Scharnowski and C. Cierpka, Exp. Fluids 52, 1629-1639 (2012)

36. Z. Wang and A. C. Bovik, IEEE Signal Proc. Lett. 9, 81-84 (2002) 